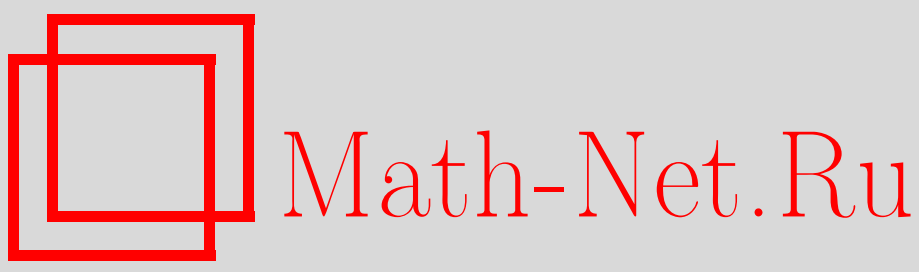

И. В. Аржанцев, Стягивания аффинных сферических многообразий, Матем. сб., 1999, том 190, номер 7, 3-22

DOI: https://doi.org/10.4213/sm413

Использование Общероссийского математического портала Math-Net.Ru подразумевает, что вы прочитали и согласны с пользовательским соглашением

http ://www.mathnet.ru/rus/agreement

Параметры загрузки:

IP : 54.162 .127 .20

26 апреля 2023 г., 14:57:43 
УДК 512.74

\author{
И.В. Аржанцев
}

\title{
Стягивания аффинных сферических многообразий
}

\begin{abstract}
В работе описан класс $G$-многообразий, которыемогут быть получены как тотальные пространства относительно известной в теории инвариантов конструкции стягивания, примененной к афффинному сферическому $G$-многообразию. Такие многообразия являются локальньми моделями для произвольных аффинных $G$-многообразий сложности один с одномерньм категорным фактором. В качестве примеров рассмотрены редуктивные алгебраические полугруппы, а также случай $G=\mathrm{SL}_{2}$.

Библиографйи: 11 названий.
\end{abstract}

1. Введение. Пусть $G$ есть связная редуктивная алгебраическая группа над алгебраически замкнутьм полем $K$ нулевой характеристики, а $Y$ - нормальное афффинное алгебраическое многообразие, на котором группа $G$ действует регулярными автоморфизмами. В работе [1] была определена конструкция стягивания действия $G: Y$ по $G$-инвариантной фильтрации $\Phi$ на алгебре $K[Y]$. Такой фильт-

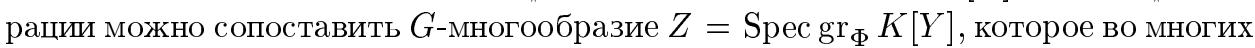
интересных случаях имеет ряд обших свойств с $G$-многообразием $Y$, но устроено несколько проще. При этом конструкция использует некоторое $\left(G \times K^{*}\right)$-многообразие $X$ (тотальное пространство стягивания), которое расслоено над прямой $\mathbb{A}^{1}$ так, что

1) слои над ненулевыми точками $G$-эквивариантно изоморфны $Y$;

2) прообраз $\mathbb{A}^{1} \backslash\{0\}$ есть прямое произведение $\left(\mathbb{A}^{1} \backslash\{0\}\right) \times Y$;

3) прообраз точки нуль есть $Z$.

В настоящей работе $\left(G \times K^{*}\right)$-многообразие $X$ со свойствами 1$)$ и 2$)$ мы будем называть $\left(G, Y, \mathbb{A}^{1}\right)$-многообразием. В п. 3 показано, что каждое $\left(G, Y, \mathbb{A}^{1}\right)$-многообразие реализуется как тотальное пространство стягивания по некоторой $G$-инвариантной фильтрации на алгебре $K[Y]$. Тем самьм $\left(G, Y, \mathbb{A}^{1}\right)$-многообразия могут быть охарактеризованы во внутренних для многообразия $Y$ терминах. Для дальнейшего хотелось бы иметь какой-либо явный способ задания $G$-инвариантных фильтраций на алгебре $K[Y]$. В п. 4 показано, что отображение $\varphi: \Xi(Y) \rightarrow \mathbb{Z}$, где $\Xi(Y)$ - спектр $G$-многообразия $Y$, удовлетворяюшее определенным условиям, задает $G$-инвариантную фильтрацию. Этим способом получается не каждая фильтрация (соответствуюший пример рассмотрен в п. 10). Однако если предположить, что действие $G: Y$ есть действие с простым спектром, то этот способ описания $G$-инвариантных фильтраций оказывается универсальным. Если дополнительно

Работа вьполнена при финансовой поддержке CRDF (грант № RM1-206) и ISSEP (грант № a98-2196).

(C) И. В. АржАнцев 1999 
потребовать, чтобы $\left(G, Y, \mathbb{A}^{1}\right)$-многообразие $X$ было нормальным, то указанное отображение $\varphi$ может быть весьма конструктивно задано с помощью определенного вьпуклого конуса (в работе названного конусом стягивания) в пространстве, натянутом на полугруппу весов $\Xi(Y) \oplus \mathbb{Z}$.

Напомним, что действие $G: Y$ (а также $G$-многообразие $Y$ ) называется $c \oint е-$ рическим, если $Y$ нормально и индуцированное действие борелевской подгруппы $B \subset G$ имеет плотную орбиту. Для аффинных $G$-многообразий сферичность эквивалентна простоте спектра алгебры регулярных функций $K[Y]$ как $G$-модуля.

Описание $\left(G, Y, \mathbb{A}^{1}\right)$-многообразий для сферического $Y$ полезно, в частности, потому, что они являются локальньпи моделями для произвольных нормальных аффинных $G$-многообразий с однопараметрическим семейством сферических орбит максимальной размерности и одномерньм категорным фактором. Пусть $X$ есть нормальное $G$-многообразие такое, что если $\pi: X \rightarrow X / / G$ - морфизм факторизации, то $X / / G \cong C$ - кривая и имеется непустое открытое подмножество $C_{0} \subset C$ такое, что $\pi^{-1}(c) \cong_{G} Y$ для всякой точки $c \in C_{0}$. Многообразие $X$ в работе [2] названо qs1-многообразием с типичным слоем $Y$. Будем говорить, что $X$ является бирачионально тривиальным, если $\pi^{-1}\left(C_{0}\right) \cong_{G} Y \times C_{0}$. В работе [2] показано, что каждое qs1-многообразие может быть получено из бирационально тривиального qs1-многообразия факторизацией по конечной группе $G$-эквивариантных автоморфизмов.

Бирационально тривиальное qs1-многообразие является $\left(G, Y, \mathbb{A}^{1}\right)$-многообразием, если $C \cong \mathbb{A}^{1}, C_{0} \cong \mathbb{A}^{1} \backslash\{0\}$ и естественное действие одномерного тора на прямой $\mathbb{A}^{1}$ продолжается до сферического действия групшы $G \times K^{*}$ на этом многообразии.

В [2] показано, что всякое бирационально тривиальное qs1-многообразие может быть получено из $\left(G, Y, \mathbb{A}^{1}\right)$-многообразий взятием обратных прообразов относительно этальных морфизмов факторов и последующей их склейкой по изоморфным открытьм подмножествам. Поэтому изучение qs1-многообразий во многих случаях может быть сведено к изучению $\left(G, Y, \mathbb{A}^{1}\right)$-многообразий. Последние являются сферическими относительно действия групшы $G \times K^{*}$ и допускают описание в терминах теории сферических вложений Луны-Вуста [3]. Использованный в настоящей работе способ описания является в известном смысле двойственным по отношению к теории Луны-Вуста. Этот способ ранее применялся в работах [4]-[6] и др. и представляется более естественньм в аффинной ситуации.

Пусть $\Xi_{+}(G)$ - полугруппа доминантных весов группы $G$. В работе [1] рассматривалось стягивание по фильтрации, определенной гомоморфизмом $h: \Xi_{+}(G) \rightarrow \mathbb{Z}_{+}$. Такую фильтрацию мы будем называть линейной. Стягивания по линейным фильтрациям интересны тем, что выполнение ряда свойств для типичного слоя $Y$ равносильно их вьполнению для особого слоя $Z$. K таким свойствам, например, относятся целозамкнутость, отсутствие нильпотентов, целостность и рациональность особенностей. Как показывают примеры, для $\left(G, Y, \mathbb{A}^{1}\right)$-многообразий на отсутствие нильпотентов и на неприводимость особого слоя $Z$ рассчитывать не приходится. Поэтому класс линейных фильтраций слишком узок для построения произвольных $\left(G, Y, \mathbb{A}^{1}\right)$-многообразий.

Мы рассматриваем более широкий класс $G$-инвариантных фильтраций, которые называем нормальнылми. Эти фильтрации описываются при помощи конусов 
стягивания, см. п. 6. Основной результат статьи (теорема 1) утверждает, что нормальные $\left(G, Y, \mathbb{A}^{1}\right)$-многообразия для сферического $G$-многообразия $Y$ с точностью до $\left(G \times K^{*}\right)$-эквивариантного изоморфизма находятся в естественном биективном соответствии с нормальньми фильтрациями на алгебре $K[Y]$. Вопрос о $G$-изоморфизме $\left(G, Y, \mathbb{A}^{1}\right)$-многообразий представляется более тонким, см. п. 12 .

В терминах конуса стягивания дан критерий приведенности особого слоя (следствие 1). Соответствующие фильтрации на $K[Y]$ естественно называть кусочнолинейными. В этих же терминах охарактеризованы неприводимые компоненты особого слоя.

В следующем пункте кратко описано, как полученные результаты переносятся на стягивания над многомерной базой $\mathbb{A}^{n}$.

В п. 11 в качестве $Y$ мы рассматриваем связную полупростую группу $G_{0}$, которая есть сферическое многообразие относительно двухстороннего $\left(G_{0} \times G_{0}\right)$-действия. Можно показать, что $\left(G_{0} \times G_{0}, G_{0}, \mathbb{A}^{1}\right)$-многообразие имеет структуру редуктивной алгебраической полугруппы. Обратно, редуктивная алгебраическая полугруппа с группой обратимых элементов $G_{0} \times K^{*}$ (кроме самой групшы $\left.G_{0} \times K^{*}\right)$ является $\left(G_{0} \times G_{0}, G_{0}, \mathbb{A}^{1}\right)$-многообразием. Таким образом, этот класс редуктивных полугрупп биективно соответствует нормальным $\left(G_{0} \times G_{0}\right)$-инвариантным фильтрациям на алгебре $K\left[G_{0}\right]$.

В последнем пункте мы описываем все возможные нормальные $\left(\mathrm{SL}_{2}, Y, \mathbb{A}^{1}\right)$-многообразия для сфферического $\mathrm{SL}_{2}$-многообразия $Y$.

Когда первоначальный вариант статьи был уже написан, Э. Б. Винберг обратил внимание автора на работу [6], где использовались аналогичные методы. После этого текст был несколько переработан с учетом результатов из [6].

Я благодарен профессору Э.Б. Винбергу за внимание к работе и помощь в оформлении текста.

2. Зафиксируем используемые обозначения.

Основное поле $K$ предполагается алгебраически замкнутым и char $K=0$;

$\mathbb{Q}$ - поле рациональных чисел;

$\mathbb{Z}_{n}-$ циклическая группа порядка $n$;

$\mathbb{A}^{n}-n$-мерное аффинное пространство над $K$;

$K^{*}$ - мультипликативная группа поля $K$ или, другими словами, одномерный тор;

$K[X]$ - алгебра регулярных функций на многообразии $X$;

Spec $A$ - афффинная схема, отвечаюшая алгебре $A$;

$A^{R}$ - подалгебра инвариантных функций алгебры $A$ с заданным действием алгебраической группы $R$;

$G$ - связная редуктивная группа над $K$;

$T \subset B$ - максимальный тор и борелевская подгруппа в $G$;

$U$ - максимальная унипотентная подгруппа в $G$;

$\Xi(G)$ - решетка весов группы $G$ относительно тора $T$;

$\Xi(G)_{+}$- полугруппа доминантных весов;

$C_{W}(G)$ - положительная камера Вейля для группы $G$ относительно фиксированной системы простых корней;

$V_{\lambda}$ - конечномерньй неприводимый $G$-модуль со старшим весом $\lambda \in \Xi(G)_{+}$. 
Для аффинного многообразия $X$ с регулярньм действием группы $G$ (далее просто $G$-многообразие) рассмотрим разложение алгебры $K[X]$ в прямую сумму $G$-подмодулей

$$
K[X]=\bigoplus_{\lambda \in \Xi(X)} W_{\lambda}
$$

где $W_{\lambda}$ - сумма всех неприводимых подмодулей из $K[X]$, изоморфных $V_{\lambda}$. Это разложение называется изотипным. Будем включать в $\Xi(X)$ только те веса $\lambda$, для которых $W_{\lambda} \neq\{0\}$. Множество весов $\Xi(X)$ есть конечно порожденная подполугруппа в полугруппе $\Xi(G)_{+}$.

Для подполугрупшы $Г$ в свободной абелевой группе обозначим через $\mathbb{Z}(\Gamma)$ минимальную подгруппу, содержащую Г.

3. Пусть $Y$ есть аффинное $G$-многообразие.

ОПрЕДЕЛЕНИЕ 1. Афринное $\left(G \times K^{*}\right)$-многообразие $X$ будем называть $\left(G, Y, \mathbb{A}^{1}\right)$-многообразием, если имеется сюръективный $K^{*}$-эквивариантный морфизм $\pi: X \rightarrow \mathbb{A}^{1}$ для эффективного действия $K^{*}: \mathbb{A}^{1}$ такой, что для каждого $a \neq 0$ имеем $\pi^{-1}(a) \cong_{G} Y$ и $\pi^{-1}\left(\mathbb{A}^{1} \backslash\{0\}\right) \cong_{\left(G \times K^{*}\right)}\left(Y \times\left(\mathbb{A}^{1} \backslash\{0\}\right)\right)$.

Напомним конструкцию стягивания действия из работы [1]. Пусть $A$ есть конечно порожденная $G$-алгебра. Предположим, что на $A$ задана какая-либо $G$-инвариантная фильтрация $\Phi$ типа $\mathbb{N}$ :

$$
1 \in A_{0} \subset A_{1} \subset \cdots \subset A_{n} \subset \cdots ; \quad A=\bigcup_{n=0}^{\infty} A_{n}, \quad A_{n} A_{m} \subset A_{n+m}
$$

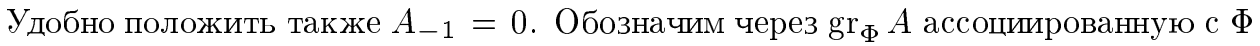
градуированную алгебру, $\operatorname{gr}_{\Phi} A=\bigoplus_{n=0}^{\infty} A_{n} / A_{n-1}$. Она является рациональной $G$-алгеброй относительно естественного действия группы $G$.

Рассмотрим теперь алгебру $A[t]$ многочленов от переменной $t$ и в ней подмножество

$$
D(\Phi)=\sum_{n=0}^{\infty} A_{n} t^{n}
$$

Из определения фильтрации следует, что $D(\Phi)$ является подалгеброй в $A[t]$, содержашей $K[t]$. Мы продолжим действие $G$ на $A$ до действия ( $K$-автоморфизмами) $G$ на $A[t]$, считая, что $t \in A[t]^{G}$. Также на алгебре $D(\Phi)$ определено действие $K^{*}$, соответствуюшее градуировке по степеням $t$. Алгебра $D(\Phi)$ не является, вообще говоря, конечно порожденной.

Пример 1 (см. рис. 1). Пусть $G=K^{*}, A=K[x]$ и $G$ действует на $A$ гомотетиями. Положим $A_{0}=K, A_{1}=A_{2}=A_{3}=\cdots=x K[x]$. В этом случае алгебра $D(\Phi)$ не конечно порождена.

Пусть теперь фильтрация $\Phi$ такова, что алгебра $D(\Phi)$ конечно порождена. Тогда имеется $G$-многообразие $\operatorname{Spec} D(\Phi)$. Морфизм $\pi: \operatorname{Spec} D(\Phi) \rightarrow \operatorname{Spec} K[t]$ является плоским, все слои этого морфизма, кроме слоя $\pi^{-1}(0), G$-эквивариантно изоморфын $\operatorname{Spec} A$, и говорят, что действие $G: \operatorname{Spec} A$ стягивается к действию 


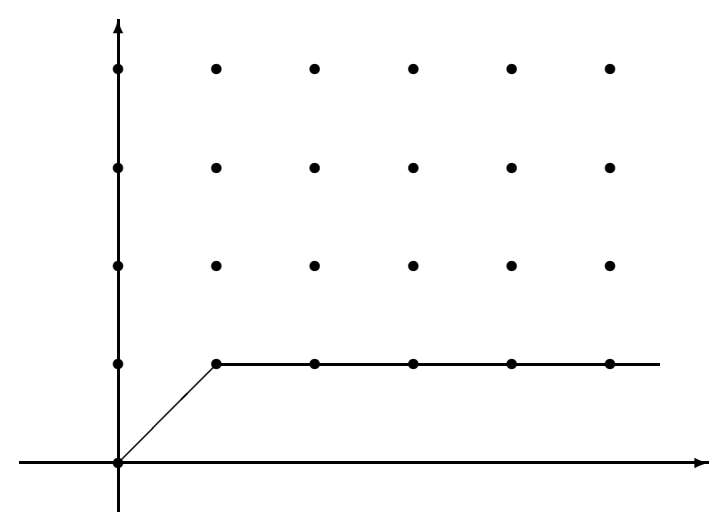

Рис. 1

$G: \pi^{-1}(0)$, а многообразие $\operatorname{Spec} D(\Phi)$ называют тотальньцм пространством стягивания.

Из предложения 9 работы [1] следует, что $\operatorname{Spec} D(\Phi)$ является $\left(G, Y, \mathbb{A}^{1}\right)$-многообразием, где $Y=\operatorname{Spec} A$. Для особого слоя $Z=\pi^{-1}(0)$ имеем $K[Z] \cong \operatorname{gr}_{\Phi} A$. Заметим, что указанная конструкция переносится на фильтрации типа $\mathbb{Z}$ с заменой алгебры $A[t]$ на алгебру $A\left[t, t^{-1}\right]$.

ПреДЛОЖЕНИЕ 1. Пусть $X$ есть $\left(G, Y, \mathbb{A}^{1}\right)$-многообразие. Тогда на алгебре $K[Y]$ имеется $G$-инвариантная фильтрация $\Phi$ такая, что многообразие $X$ $\left(G \times K^{*}\right)$-изоморфно тотальному пространству стягивания действия $G: Y$ по фильтрации $\Phi$.

ДоказАтельство. Рассмотрим изотипное разложение

$$
K[Y]=\bigoplus_{\lambda \in \Xi(Y)} W_{\lambda} .
$$

Имеет место изоморфизм $K\left[\pi^{-1}\left(\mathbb{A}^{1} \backslash\{0\}\right)\right] \cong K[Y] \otimes_{K} K\left[t, t^{-1}\right]$, где $t-$ координата на прямой $\mathbb{A}^{1}$. Здесь изотипными компонентами относительно действия группы $G \times K^{*}$ являются подпространства $W_{\lambda} \otimes_{K} K t^{i}, i \in \mathbb{Z}$. Включение $K[X] \subset K\left[\pi^{-1}\left(\mathbb{A}^{1} \backslash\{0\}\right)\right]$ показывает, что если

$$
K[X]=\bigoplus_{(\lambda, i) \in \Xi(Y) \oplus \mathbb{Z}} U_{(\lambda, i)}
$$

есть $\left(G \times K^{*}\right)$-изотипное разложение алгебры $K[X]$, то $U_{(\lambda, i)} \subset W_{\lambda} \otimes_{K} K t^{i}$, и потому $U_{(\lambda, i)}$ может быть отождествлено с подпространством в $W_{\lambda}$. Обозначим это подпространство через $U_{(\lambda, i)}^{\prime}$. Тогда $U_{(\lambda, i)}^{\prime} \subset U_{(\lambda, i+1)}^{\prime}$ и $W_{\lambda}$ совпадает с объединением подпространств $U_{(\lambda, i)}^{\prime}$.

Определим $G$-инвариантную фильтрацию на $K[Y]$

$$
\cdots \subset K[Y]_{-i} \subset \cdots \subset K[Y]_{0} \subset K[Y]_{1} \subset \cdots \subset K[Y]_{i} \subset \cdots
$$

по формуле $K[Y]_{i}=\bigoplus_{\lambda} U_{(\lambda, i)}^{\prime}$. 
Очевидно, что свойство $K[Y]=\bigcup_{i=-\infty}^{\infty} K[Y]_{i}$ здесь выполнено. Остается проверить, что $K[Y]_{i} K[Y]_{j} \subset K[Y]_{i+j}$. Это следует из того, что $U_{\left(\lambda_{1}, i\right)} U_{\left(\lambda_{2}, j\right)} \subset$ $\sum_{\lambda} U_{(\lambda, i+j)}$.

4. Следуя В. Л. Попову, можно задать $G$-инвариантную фильтрацию $\Phi(\varphi)$ на алгебре $K[Y]$ отображением $\varphi: \Xi(Y) \rightarrow \mathbb{Z}$, положив $K[Y]_{i}=\bigoplus_{\lambda \in \Xi(Y), \varphi(\lambda) \leqslant i} W_{\lambda}$.

При этом на отображение $\varphi$ нужно наложить определенные ограничения. $\mathrm{У}_{\mathrm{M}-}$ ножение в алгебре $K[Y]$ позволяет рассматривать произведение $W_{\lambda} W_{\mu}$ как подмодуль в $K[Y]$ для любых $\lambda, \mu \in \Xi(Y)$. Определим набор $T_{Y}(\lambda, \mu)$ как множество тех весов $\varepsilon \in \Xi(Y)$, для которых $W_{\lambda} W_{\mu}$ пересекается с $W_{\varepsilon}$ по ненулевому подпространству. Известно, что множество $T_{Y}(\lambda, \mu)$ конечно, содержит вес $\lambda+\mu$ и все прочие веса этого множества получаются из $\lambda+\mu$ вычитанием некоторой линейной комбинации простых корней с цельми неотрицательными коэффициентами.

Условие $K[Y]_{i} K[Y]_{j} \subset K[Y]_{i+j}$ из определения фильтрации равносильно условию

(A) $\varphi(\varepsilon) \leqslant \varphi(\lambda)+\varphi(\mu)$ для всех $\lambda, \mu \in \Xi(Y)$ и всех $\varepsilon \in T_{Y}(\lambda, \mu)$.

Остальные требования из определения фильтрации выполнены автоматически. В работе [1] в качестве отображения $\varphi$ рассматривалось ограничение на $\Xi(Y)$ аддитивного гомоморфизма $h: \Xi_{+}(G) \rightarrow \mathbb{Z}_{+}$такого, что после продолжения его до линейной формы на $\Xi(G) \otimes_{\mathbb{Z}} \mathbb{Q}$ значения этой формы на простых корнях положительны (существование такого гомоморфизма несложно установить). Для этого отображения условие (А) выполнено. Фильтрацию, определенную таким гомоморфизмом, будем называть линейной.

Если каждый из $G$-модулей $W_{\lambda}$ является неприводимым (алгебра $K[Y]$ имеет простой спектр), то для любого $\lambda$ и для любой $G$-инвариантной фильтрации $\Phi$ найдется такой номер $i$, что $W_{\lambda} \cap K[Y]_{i-1}=\{0\}$ и $W_{\lambda} \subset K[Y]_{i}$. Поэтому здесь любая $G$-инвариантная фильтрация на $K[Y]$ задается некоторым отображением $\varphi: \Xi(Y) \rightarrow \mathbb{Z}$, а именно

$$
\varphi(\lambda)=\min _{W_{\lambda} \subset K[Y]_{i}} i .
$$

Для произвольной $G$-алгебры это не так, соответствующий пример рассмотрен в п. 10.

5. Напомним в этом разделе некоторые известные факты из теории инвариантов. Пусть $X$ есть неприводимое аффинное $G$-многообразие. Нормальность многообразия $X$ по определению означает, что алгебра $K[X]$ целозамкнута в своем поле частных.

КритЕРИЙ Луны-ВУСта (см., например, [7; гл. 3]). Аффинное $G$-многообразие $X$ нормально тогда и только тогда, когда алгебра $K[X]^{U}$ челозамкнута.

Говорят, что действие $G: X$ есть действие с простым спектром, если каждая изотипная компонента $W_{\lambda}$ алгебры $K[X]$ является неприводимым $G$-модулем.

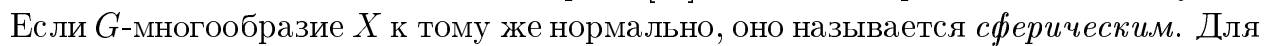
действий с простым спектром алгебра $K[X]^{U}$ имеет простой спектр относительно действия максимального тора, и потому $X_{U}=\operatorname{Spec~} K[X]^{U}$ есть торическое многообразие. Из теории торических многообразий известно, что нормальность $X_{U}$ 
эквивалентна условию насьшенности полугрупшы весов $\Xi\left(X_{U}\right)$ тора $T$, т.е. если $\varepsilon \in \mathbb{Z}\left(\Xi\left(X_{U}\right)\right)$ и $n \varepsilon \in \Xi\left(X_{U}\right)$ для некоторого $n>0$, то $\varepsilon \in \Xi\left(X_{U}\right)$. Насышенные полугруппы - это в точности полугрупшы, совпадающие с пересечением решетки $\mathbb{Z}\left(\Xi\left(X_{U}\right)\right)$ и некоторого выпуклого конуса в пространстве $\mathbb{Z}\left(\Xi\left(X_{U}\right)\right) \otimes_{\mathbb{Z}} \mathbb{Q}$. Полугруппа $\Xi\left(X_{U}\right)$ естественно отождествляется с полугруппой $\Xi(X)$. Окончательно получаем

ПРЕДЛОЖЕНИЕ 2. Аффинное $G$-многообразие $X$ с простым спектром нормально тогда и только тогда, когда полугруппа $\Xi(X)$ совпадает с пересечением решетки $\mathbb{Z}(\Xi(X))$ и некоторого выпуклого конуса в пространстве $\mathbb{Z}(\Xi(X)) \otimes_{\mathbb{Z}} \mathbb{Q}$.

Этим предложением мы будем неоднократно пользоваться. Заметим также, что полугруппа из предложения 2 конечно порождена по лемме Гордана, см., например, [8].

6. Пусть $Y$ есть сферическое $G$-многообразие, а $X$ есть $\left(G, Y, \mathbb{A}^{1}\right)$-многообразие, которое всюду далее будет предполагаться нормальным. Алгебра регулярных функций $K[X]$ естественно вкладывается в алгебру $K[Y] \otimes_{K} K\left[\mathbb{A}^{1} \backslash\{0\}\right]$. Последняя алгебра имеет простой спектр относительно действия группы $G \times K^{*}$, и потому $\Xi(X) \subset \Xi(Y) \oplus \mathbb{Z}$.

Обозначим $N=\mathbb{Z}(\Xi(Y) \oplus \mathbb{Z}) \otimes_{\mathbb{Z}} \mathbb{Q}$ и $W=\mathbb{Z}(\Xi(Y)) \otimes_{\mathbb{Z}} \mathbb{Q}$ - гиперплоскость в $N$.

Лемма 1. а) Полугруппа $\Xi(X)$ совпадает с пересечением некоторого въпуклого конуса $C(X)$ максимальной размерности в пространстве $N$ и полугруппьь $\Xi(Y) \oplus \mathbb{Z}$.

b) Конус $C(X)$ содержит луч $V=(0, a) \in N, a \geqslant 0$, и не содержит луч $-V$.

c) При проекции на $W$ параллельно лучу $V$ образ конуса $C(X)$ совпадает $c$ въпуклой оболочкой $\Xi(Y)$.

ДокАЗАтЕльство. а) Следует из предложения 2.

b) Из сферичности действия $G: Y$ вытекает наличие в $Y$ плотной $G$-орбиты. Согласно определению $\left(G, Y, \mathbb{A}^{1}\right)$-многообразия алгебра $K[X]^{G}$ есть алгебра многочленов от одной переменной и ее образуюшая имеет вес $(0,1)$.

Для морфизма $\pi: X \rightarrow \mathbb{A}^{1}$ прообраз ненулевой точки прямой $\mathbb{A}^{1} G$-изоморфен многообразию $Y$. Отсюда следует утверждение с).

Рассмотрим функцию $\varphi_{X}: \Xi(Y) \rightarrow \mathbb{Z}$, заданную формулой

$$
\varphi_{X}(\lambda)=\min _{(\lambda, a) \in \Xi(X)} a .
$$

Если $\varepsilon \in T_{Y}(\lambda, \mu)$ и $(\lambda, a) \in \Xi(X),(\mu, b) \in \Xi(X)$, то $(\varepsilon, a+b) \in \Xi(X)$. Отсюда

$$
\varphi_{X}(\varepsilon) \leqslant \varphi_{X}(\lambda)+\varphi_{X}(\mu) \text { для каждого } \varepsilon \in T_{Y}(\lambda, \mu) .
$$

Для выпуклого конуса $C$ в пространстве $N$, удовлетворяющего условиям b) и с) предыдущей леммы, определим функцию $\varphi_{C}: \Xi(Y) \rightarrow \mathbb{Z}$ :

$$
\varphi_{C}(\lambda)=\min _{(\lambda, a) \in C, a \in \mathbb{Z}} a
$$

Ясно, что функции $\varphi_{X}$ и $\varphi_{C(X)}$ совпадают. 
ОПРЕДЕЛЕНИЕ 2. Назовем выпуклый конус $C$ в пространстве $N$ конусом гивания, если выполнены следующие условия:

1) конус $C$ содержит луч $V$ и не содержит луч $-V$;

2) при проекции на гиперплоскость $W$ параллельно лучу $V$ образ конуса $C$ совпадает с выпуклой оболочкой $\Xi(Y)$;

3) $\varphi_{C}(\varepsilon) \leqslant \varphi_{C}(\lambda)+\varphi_{C}(\mu)$ для каждого $\varepsilon \in T_{Y}(\lambda, \mu)$.

ПРЕДЛОЖЕНИЕ 3. Нормальнье $\left(G, Y, \mathbb{A}^{1}\right)$-многообразия с точностью до $\left(G \times K^{*}\right)$-изоморфизма биективно соответствуют конусам стягивания в пространстве $N$.

ДоказАТЕльство. $\left(G, Y, \mathbb{A}^{1}\right)$-многообразию $X$ сопоставляется конус стягивания $C(X)$. Обратно, для конуса стягивания $C$ рассмотрим подпространство $L(C)$ в алгебре $K[Y] \otimes_{K} K\left[\mathbb{A}^{1} \backslash\{0\}\right]$, состоящее из тех неприводимых $\left(G \times K^{*}\right)$-модулей, старшие веса которых принадлежат конусу $C$. Умножение в алгебре $K[Y] \otimes_{K}$ $K\left[\mathbb{A}^{1} \backslash\{0\}\right]$ индуцирует на этом подпространстве структуру подалгебры, что обеспечивается условием 3 ). Известно, что для произвольной рациональной $G$-алгебры $A$ конечная порожденность $A$ эквивалентна конечной порожденности $A^{U}$, см. [1]. Для алгебры $L(C)$ конечная порожденность $L(C)^{U}$ следует из леммы Гордана. Поэтому алгебра $L(C)$ конечно порождена. Из условий 1$)$ и 2) следует, что $L(C)^{G}$ есть алгебра многочленов от одной переменной, и для морфизма факторизации $\pi: \operatorname{Spec} L(C) \rightarrow \mathbb{A}^{1}$ мы имеем $\pi^{-1}\left(\mathbb{A}^{1} \backslash\{0\}\right) \cong Y \times\left(\mathbb{A}^{1} \backslash\{0\}\right)$. Следовательно, $\operatorname{Spec} L(C)$ есть $\left(G, Y, \mathbb{A}^{1}\right)$-многообразие. В силу различия $\left(G \times K^{*}\right)$-модульной структуры в алгебре регулярных функций различньп конусам отвечают различные $\left(G, Y, \mathbb{A}^{1}\right)$-многообразия.

Хотелось бы выяснить, для каких конусов $C$ в пространстве $N$ с условиями 1) и 2 ) из определения 2 выполнено также условие 3 ). Это условие есть условие “отрицательности” на простых корнях группы $G$. Заметим, что пространство $N$ можно рассматривать как подпространство в $N(G)=\mathbb{Z}(\Xi(G) \oplus \mathbb{Z}) \otimes_{\mathbb{Z}} \mathbb{Q}$. Поэтому несложно проверить, что для выполнения условия 3) достаточно выполнения следующего условия:

(В) найдется выпукльй конус $\widehat{C}$ в пространстве $N(G)$ такой, что $N \cap \widehat{C} \cap$ $C_{W}\left(G \times K^{*}\right)=C$ и отрицательные корни группы $G$ принадлежат $\widehat{C}$.

Если ранг решетки $\Xi(X)$ совпадает с рангом решетки $\Xi(G)$, то в качестве $\widehat{C}$ можно рассматривать максимальный конус в $N(G)$, пересечение которого с $C_{W}\left(G \times K^{*}\right)$ совпадает с $C$ (см. [6; теорема 2]).

Будем говорить, что сферическое $G$-многообразие $Y$ есть многообразие типа I, если для каждого конуса стягивания условие (В) выполнено. В противном случае $G$-многообразие соответствует типу II. Для выяснения типа $G$-многообразия необходимо иметь некоторую информацию о множествах $T_{Y}(\lambda, \mu)$. K типу I относятся группа $G$ как однородное $(G \times G)$-пространство относительно двухстороннего действия, см. [6] или п. 11, и однородные пространства $\mathrm{SL}_{2} / N$ и $\mathrm{SL}_{2} / T$, см. п. 12. K типу II относятся все торические и, более общо, все $S$-многообразия в терминологии работы [4], т.е. $G$-многообразия, стабилизатор каждой точки на которых содержит некоторую максимальную унипотентную подгруппу $U$ группы $G$. В этой 
ситуации условие 3 ) выполнено для любого конуса, так как $S$-многообразия характеризуются условием

(C) $T_{Y}(\lambda, \mu)=\{\lambda+\mu\}$ для всех $\lambda, \mu \in \Xi(Y)$.

В самом деле, нормальное сферическое $G$-многообразие $Y$ является $S$-многообразием тогда и только тогда, когда алгебра регулярных функций $K[Y]$ реализуется как левоинвариантная подалгебра алгебры $S$ регулярных функций на группе $G$, правоинвариантных относительно максимальной унипотентной подгрупшы, см. [4]. Из теоремы 5 работы [1] следует, что последнее условие эквивалентно условию (C).

7. Вернемся к описанию $G$-инвариантных фильтраций на алгебре $K[Y]$ в случае, когда $Y$ есть сферическое $G$-многообразие. Потребуем, чтобы соответствующая фильтрации $\Phi$ алгебра $D(\Phi)$ была конечно порождена и целозамкнута. Оказывается, что при этих ограничениях все такие фильтрации могут быть эффективно описаны при помощи конусов стягивания. Действительно, мы имеем нормальное $\left(G, Y, \mathbb{A}^{1}\right)$-многообразие $X=\operatorname{Spec} D(\Phi)$, и соответствующая ему функция $\varphi_{X}$ определяет некоторую $G$-инвариантую фильтрацию на $K[Y]$ (ср. условие $(\mathrm{A})$ и свойство $(* *))$. Из равенства $\varphi_{X}=\varphi_{C(X)}$ следует, что получаемые так фильтрации характеризуются следующим определением.

ОпРЕДЕЛЕниЕ 3. $G$-инвариантная фильтрация $\Phi$ на алгебре $K[Y]$ называется нормальной, если найдется конечный набор линейных функций $f_{i}: \mathbb{Z}(\Xi(Y)) \otimes_{\mathbb{Z}} \mathbb{Q}$ $\rightarrow \mathbb{Q}$ такой, что $\varphi_{\Phi}(\lambda)=\min a$, где $a \in \mathbb{Z}$ и $a \geqslant f_{i}(\lambda)$ для всех значений $i$.

Из формулы $(*)$ и определения функции $\varphi_{X}$ следует, что функция $\varphi_{X}$ задает именно исходную фильтрацию $\Phi$. Обратно, каждому нормальному $\left(G, Y, \mathbb{A}^{1}\right)$ многообразию отвечает $G$-инвариантная фильтрация $\Phi$ (предложение 1 ), эта фильтрация определяется функцией $\varphi_{C(X)}$ и потому является нормальной. Используя предложение 3 , окончательно получаем следующую теорему.

Теорема 1. Пусть $Y$ есть аффинное сферическое $G$-многообразие. Тогда нормальные $\left(G, Y, \mathbb{A}^{1}\right)$-многообразия биективно соответствуют нормальным фильтрациям на алгебре $K[Y]$.

8. Далее мы изучим некоторые свойства особого слоя $\left(G, Y, \mathbb{A}^{1}\right)$-многообразия $X$ в терминах конуса стягивания $C=C(X)$. Для этого нам понадобятся следующие обозначения. Пусть $\partial C$ есть граница конуса $C$, т.е. объединение всех его собственных граней. Положим $\Xi(Y)_{\partial C}=\left\{\lambda \in \Xi(Y):\left(\lambda, \varphi_{C}(\lambda)\right) \in \partial C\right\}$. Для каждой алгебры $A$ пусть $A_{\text {red }}$ есть факторалгебра $A$ по максимальному нильпотентному идеалу и $(\operatorname{Spec} A)_{\mathrm{red}}=\operatorname{Spec} A_{\mathrm{red}}$. Согласно [9; предложение 3.26$] G$-модульная структура $K\left[\pi^{-1}(0)\right]$ совпадает с $G$-модульной структурой $K[Y]$.

ЛЕмма 2. Имеем G-модульное разложсение

$$
K\left[\pi^{-1}(0)\right]_{\mathrm{red}}=\bigoplus_{\lambda \in \Xi(Y)_{\partial C}} V_{\lambda}
$$


ДокАЗАТЕЛЬСтво. Несложно понять, что следующие условия эквивалентны:

1) старший вектор неприводимого $G$-модуля $V_{\lambda}$ является нильпотентом при сужении на особьй слой;

2) некоторая степень старшего вектора модуля $V_{\lambda} \subset K\left[\pi^{-1}(0)\right]$ делится в алгебре $D(\Phi)$ на переменную $t$ (см. определение стягивания);

3) сушествует $n>0$ такое, что $\varphi_{C}(n \lambda)<n \varphi_{C}(\lambda)$;

4) $\lambda \notin \Xi(Y) \partial C$.

Назовем конус стягивания $C$ иелочисленным, если $\Xi(Y)=\Xi(Y)$ дс. Это условие равносильно тому, что линейные функции $f_{i}$ из определения 3 принимают целые значения на элементах полугруппы $\Xi(Y)$.

СЛЕДСТВИЕ 1. Особъй слой тотального пространства стягивания приведен тогда и только тогда, когда конус стягивания целочислен.

Перейдем к рассмотрению неприводимых компонент особого слоя.

Собственную грань максимальной размерности конуса стягивания назовем $m$-гранью, если она не содержит луч $V$. Будем говорить, что вес $\lambda \in \Xi(Y)$ принадлежит $m$-грани $F$ конуса стягивания $C$ и условно записьвать $\lambda \in F$, если $\left(\lambda, \varphi_{C}(\lambda)\right) \in F$.

ПРЕДЛОЖЕНИЕ 4. Имеется естественное биективное соответствие межсду неприводимыци компонентами особого слоя $\left(G, Y, \mathbb{A}^{1}\right)$-многообразия $X$ и т-гранями конуса стягивания $C(X)$. В частности, особъй слой неприводим тогда и только тогда, когда т-грань единственна.

ДокаЗАТЕЛьство. Пусть $C_{1}, C_{2}, \ldots, C_{k}$ - неприводимые компоненты особого слоя и $I\left(C_{1}\right), I\left(C_{2}\right), \ldots, I\left(C_{k}\right)$ - соответствующие простые идеалы в алгебре $K\left[\pi^{-1}(0)\right]_{\mathrm{red}}$. Тогда для любого $i$ имеется однозначно определенное $G$-модульное разложение $K\left[\pi^{-1}(0)\right]=I\left(C_{i}\right) \oplus P\left(C_{i}\right)$. Пространство $P\left(C_{i}\right) \quad G$-изоморфно $K\left[C_{i}\right]_{\mathrm{red}}$. Пусть

$$
P\left(C_{i}\right)=\bigoplus_{\lambda \in \Xi\left(C_{i}\right)} V_{\lambda} .
$$

Если веса $\lambda^{\prime}, \lambda^{\prime \prime} \in \partial C$ не принадлежат одной собственной грани максимальной размерности конуса $C$, то $\lambda^{\prime}+\lambda^{\prime \prime} \notin \partial C$ и для соответствуюших старших векторов имеем $f_{\lambda^{\prime}} f_{\lambda^{\prime \prime}}=0$ в алгебре $K\left[\pi^{-1}(0)\right]_{\mathrm{red}}$. Это показывает, что все веса полугруппы $\Xi\left(C_{i}\right)$ лежат на одной $m$-грани.

Предположим, что $m$-грани $F$ соответствуют несколько компонент $C_{i_{1}}, \ldots, C_{i_{l}}$. Тогда для каждого $i_{p}$ найдется такой вес $\lambda\left(i_{p}\right)$, что $\lambda\left(i_{p}\right) \in F \backslash \Xi\left(C_{i_{p}}\right)$ и потому старший вектор $f_{\lambda\left(i_{p}\right)}$ принадлежит $I\left(C_{i_{p}}\right)$. Произведение старших векторов $f_{\lambda\left(i_{1}\right)} f_{\lambda\left(i_{2}\right)} \cdots f_{\lambda\left(i_{k}\right)}$ равно нулю на каждой компоненте особого слоя, тогда как $\lambda\left(i_{1}\right)+\lambda\left(i_{2}\right)+\cdots+\lambda\left(i_{k}\right) \in F$. Полученное противоречие завершает доказательство.

ЗАмЕчАниЕ 1. У конуса стягивания, отвечающего линейной фильтрации, есть только одна $m$-грань - это график гомоморфизма. 
СлЕДСТвИЕ 2. Если $\lambda_{1} \in \partial C \backslash F \quad u \quad \lambda_{2} \in \partial C$, mo $\mu \notin F$ для каждого $\mu \in T_{Y}\left(\lambda_{1}, \lambda_{2}\right)$.

Доказательство следует из того факта, что для компоненты $C_{F}$, отвечающей $m$-грани $F$,

$$
I\left(C_{F}\right)=\bigoplus_{\lambda \in \partial C \backslash F} V_{\lambda}
$$

является идеалом.

СлЕДСТВИЕ 3. Имеет место равенство

$$
T_{C_{F}}\left(\lambda_{1}, \lambda_{2}\right)=T_{Y}\left(\lambda_{1}, \lambda_{2}\right) \cap F
$$

Из [10], [11] следует, что каждая компонента особого слоя является нормальным сферическим $G$-многообразием. Из условия $(\mathrm{C})$ вытекает

СлеДСтвИЕ 4. Компонента $C_{F}$ особого слоя на $\left(G, Y, \mathbb{A}^{1}\right)$-многообразии является $S$-многообразием тогда и только тогда, когда $T_{Y}\left(\lambda_{1}, \lambda_{2}\right) \cap F=$ $\left\{\lambda_{1}+\lambda_{2}\right\}$ для всех $\lambda_{1}, \lambda_{2} \in F$.

Для вьполнения этого условия достаточно, чтобы существовала линейная форма на $\Xi(G) \otimes_{\mathbb{Z}} \mathbb{Q}$, отрицательная на простых корнях, график которой над $W$ содержал быгрань $F$. Так для линейных стягиваний особый слой является нормальным $S$-многообразием.

Теорему 7 из работы [1] можно дополнить следующим образом.

ПреДЛОЖЕНИЕ 5. Пусть $A$ - рациональная конечно порождденная $G$-алгебра с простым спектром. Для линейных фильтрачий следующие условия эквивалентнь:

1) алгебра А челозамкнута;

2) алгебра $\operatorname{gr}_{\Phi} A$ челозамкнута;

3) алгебра $D(\Phi)$ челозамкнута.

$B$ этой ситуации многообразия $\operatorname{Spec} A, \operatorname{Spec}_{\Phi} A$ и $\operatorname{Spec} D(\Phi)$ имеют рациональные особенности.

ДокаЗАТЕльство. Эквивалентность 1) и 2) доказана в [1].

Если $A$ конечно порождена и целозамкнута, то из предложения 2 и леммы Гордана следует конечная порожденность и целозамкнутость $D(\Phi)$.

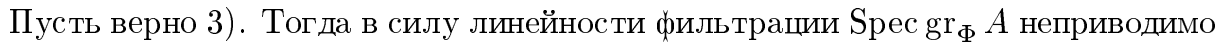
и нормально, см. [11].

Наконец, особенности сферических многообразий рациональны [1], а $\left(G, Y, \mathbb{A}^{1}\right)$ многообразие сферично относительно действия группы $G \times K^{*}$.

9. Можно рассматривать многообразия, аналогичные многообразиям из определения 1 , над многомерной базой. Большинство полученных выше результатов распространяется и на эту ситуацию. 
ОПРЕДЕЛЕНИЕ 4. Аффинное $\left(G \times\left(K^{*}\right)^{n}\right)$-многообразие $X$ будем называть $\left(G, Y, \mathbb{A}^{n}\right)$-многообразием, если имеется сюръективный $\left(K^{*}\right)^{n}$-эквивариантньй морфизм $\pi: X \rightarrow \mathbb{A}^{n}$ для естественного эффективного действия $\left(K^{*}\right)^{n}: \mathbb{A}^{n}$ такой, что для каждого $a \in\left(\mathbb{A}^{1} \backslash\{0\}\right)^{n}$ имеем

$$
\pi^{-1}(a) \cong_{G} Y \text { и } \pi^{-1}\left(\left(\mathbb{A}^{1} \backslash\{0\}\right)^{n}\right) \cong_{\left(G \times\left(K^{*}\right)^{n}\right)}\left(Y \times\left(\left(\mathbb{A}^{1} \backslash\{0\}\right)^{n}\right)\right) .
$$

Если $Y$ - сферическое $G$-многообразие, то $\Xi(X) \subset \Xi(Y) \oplus \mathbb{Z}^{n}$ и полугруппа $\Xi(X)$ получается пересечением в пространстве $W \oplus \mathbb{Q}^{n}$ некоторого вьпуклого конуса $C(X)$, содержащего координатные лучи $V_{1}, \ldots, V_{n}$ и не содержащего $-V_{1}, \ldots,-V_{n}$, и полугруппы $\Xi(Y) \oplus \mathbb{Z}^{n}$.

Конус $C(X)$ можно считать конусом многомерного стягивания. Условие 3$)$ из определения 2 будет иметь вид: если $(\lambda, u) \in \Xi(Y) \oplus \mathbb{Z}^{n}$ и $(\mu, w) \in \Xi(Y) \oplus \mathbb{Z}^{n}$ лежат в конусе $C(X)$, то и $(\varepsilon, u+w) \in C(X)$ для каждого $\varepsilon \in T_{Y}(\lambda, \mu)$.

В алгебре $K[Y]\left[t_{1}, t_{1}^{-1}, \ldots, t_{n}, t_{n}^{-1}\right]$ можно задать подалгебру $D$, порожденную элементами

$$
f t_{1}^{i_{1}} \cdots t_{n}^{i_{n}}, \quad f \in K[Y]_{\lambda}, \quad\left(\lambda, i_{1}, \ldots, i_{n}\right) \in C(X)
$$

и тогда $X=\operatorname{Spec} D$.

Определим понятие фильтрации типа $\mathbb{Z}^{n}$ на алгебре $K[Y]$. Зафиксируем следующие обозначения. Для двух элементов $s=\left(i_{1}, \ldots, i_{n}\right) \in \mathbb{Z}^{n}$ и $s^{\prime}=\left(i_{1}^{\prime}, \ldots, i_{n}^{\prime}\right) \in$ $\mathbb{Z}^{n}$ будем говорить, что $s \leqslant s^{\prime}$, если $i_{k} \leqslant i_{k}^{\prime}$ для любого $k$, и что $s<s^{\prime}$, если хотя бы одно из этих неравенств является строгим. Назовем $G$-инвариантной фuльтрацией на $G$-алгебре $A$ такой набор $G$-инвариантных подпространств $A_{s}, s \in \mathbb{Z}^{n}$, что

1) $A_{s} \subset A_{s^{\prime}}$ для любых $s \leqslant s^{\prime}$;

2) $A=\bigcup_{s \in \mathbb{Z}^{n}} A_{s}$;

3) $A_{s} A_{s^{\prime}} \subset A_{s+s^{\prime}}$ для любых $s, s^{\prime} \in \mathbb{Z}^{n}$.

В случае произвольного аффинного $G$-многообразия $Y$ для каждого $\left(G, Y, \mathbb{A}^{n}\right)$ многообразия $X$ можно определить $G$-инвариантную $\mathbb{Z}^{n}$-фильтрацию на алгебре $K[Y]$ и доказать аналог предложения 1 . В случае, когда многообразие $Y$ сферично, определяемые конусами многомерного стягивания $\mathbb{Z}^{n}$-фильтрации будем, как и выше, называть нормальнымми, и также получаем взаимно однозначное соответствие между $\left(G, Y, \mathbb{A}^{n}\right)$-многообразиями (с точностью до $\left(G \times\left(K^{*}\right)^{n}\right)$-изоморфизма) и нормальными $\mathbb{Z}^{n}$-фильтрациями на алгебре $K[Y]$.

Например, для $G=\mathrm{SL}_{2}, n=2$ будем откладывать точки из $\Xi(Y)$ по оси $O x$ и рассмотрим конус многомерного стягивания в $\mathbb{Q}^{3}$, натянутый на лучи $O y, O z$, $\{z=0, x=y\}$ и $\{y=0, x=z\}$. Сечение этого конуса плоскостью $\{x=1\}$ изображено на рис. 2.

Пусть $\varepsilon$ - фундаментальный вес групшы $\mathrm{SL}_{2}$. Рис. 2 показывает, что изотипная компонента $W_{\varepsilon}$ алгебры $K[Y]$ принадлежит как $K[Y]_{(1,0)}$, так и $K[Y]_{(0,1)}$.

Для $\left(G, Y, \mathbb{A}^{n}\right)$-многообразия $X$ алгебра регулярных функций на слое $Z$ над точкой $0 \in \mathbb{A}^{n}$ описывается формулой

$$
K[Z]=\operatorname{gr} K[Y]=\bigoplus_{s \in \mathbb{Z}^{n}} K[Y]_{s} / K[Y]_{s}^{\prime},
$$

где $K[Y]_{s}^{\prime}=\bigoplus_{s^{\prime}<s} K[Y]_{s^{\prime}}$ 

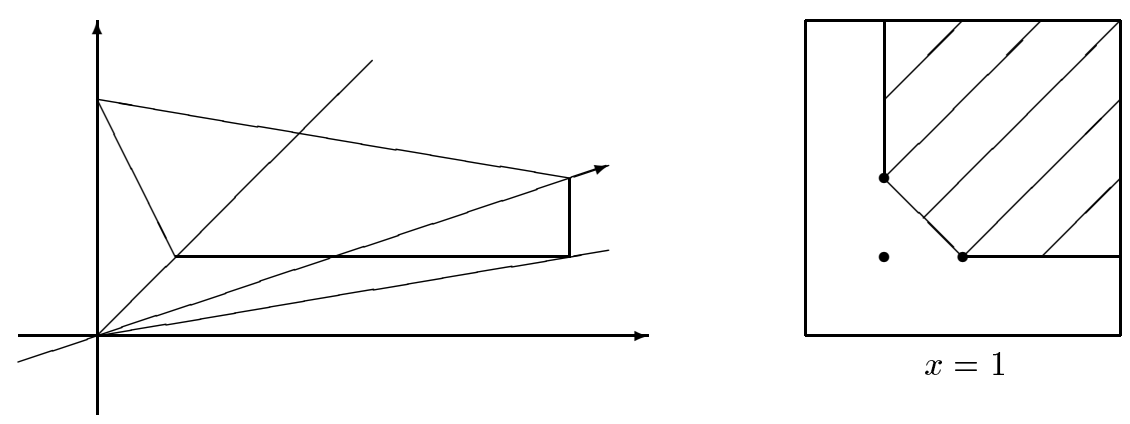

Рис. 2

10. Если не предполагать, что $Y$ является сферическим $G$-многообразием, то не каждая $G$-инвариантная $\mathbb{Z}$-фильтрация на алгебре $K[Y]$ задается некоторым отображением $\varphi: \Xi(Y) \rightarrow \mathbb{Z}$.

Приведем конкретный пример. Предположим, что многообразие $Y$ и $\left(G, Y, \mathbb{A}^{1}\right)$ многообразие $X$ нормальны и соответствуюшая $X$ фильтрация определяется отображением $\varphi: \Xi(Y) \rightarrow \mathbb{Z}$. Тогда из критерия Луны-Вуста следует, что полугруппа $\Xi(X)=\{(\lambda, i): \varphi(\lambda) \leqslant i\}$ в пространстве $N=\mathbb{Z}(\Xi(Y) \oplus \mathbb{Z}) \otimes_{\mathbb{Z}} \mathbb{Q}$ совпадает с пересечением некоторого вьпуклого конуса $C$ и решетки $\mathbb{Z}(\Xi(X))$. При этом конус $C$ удовлетворяет условиям 1)-3) определения 2 .

Пусть $G=\mathrm{SL}_{2}$. Тогда $\operatorname{rk} G=1$ и у конуса $C$ имеется всего одна $m$-грань. Алгебpa $U$-инвариантов на особом слое получается факторизацией алгебры $U$-инвариантов на $Y$ по конечной циклической группе и потому сохраняет целозамкнутость. Таким образом, особый слой нормален.

Пусть $Y=\mathrm{SL}_{2} / \mathbb{Z}_{3}$, где $\mathbb{Z}_{3}=\left\{\left(\begin{array}{cc}\alpha & 0 \\ 0 & \alpha^{-1}\end{array}\right): \alpha \in K, \alpha^{3}=1\right\}$. Рассмотрим естественное действие группы $\mathrm{SL}_{2}$ в пространстве бинарных форм степени три $V_{3}=\left\{a x^{3}+b x^{2} y+c x y^{2}+d y^{3}\right\}$. Образуюшая $F$ алгебры инвариантов для такого действия - дискриминант формы - есть многочлен степени четыре (его явный вид указан в [ $4 ;$ п. 012]). Рассмотрим $\mathrm{SL}_{2}$-модуль $V_{3} \oplus \mathbb{A}^{1}$ с тривиальньм $\mathrm{SL}_{2}$-действием на втором множителе и в нем подмногообразие

$$
X=\left\{(\psi, z): \psi \in V_{3}, z \in \mathbb{A}^{1}, z^{4}=F(\psi)\right\} .
$$

Лемма 3. Многообразие $X$ есть $\left(\mathrm{SL}_{2}, \mathrm{SL}_{2} / \mathbb{Z}_{3}, \mathbb{A}^{1}\right)$-многообразие.

ДокАЗАТЕЛЬСтво. Орбита бинарной формы третьей степени с ненулевьм дискриминантом пересекает прямую $\left\langle x^{3}+y^{3}\right\rangle$. Поэтому орбита общего положения на $V_{3}$ (и на $X$ ) замкнута и изоморфна $\mathrm{SL}_{2} / \mathbb{Z}_{3}$. Многообразие $X$ есть гиперповерхность в $\mathbb{A}^{5}$, заданная уравнением $z^{4}=F(\psi)$. Множество особенностей этой гиперповерхности есть собственное подмногообразие в множестве $\{z=0\}$. Следовательно, $X$ неособо в коразмерности один, а для гиперповерхности это эквивалентно нормальности. Алгебра инвариантов $K[X]^{\mathrm{SL}_{2}}$ есть $K[z]$. Остается проверить условие $X \backslash\{z=0\} \cong \mathrm{SL}_{2} / \mathbb{Z}_{3} \times K^{*}$ или $K[X]_{(z)} \cong K\left[\mathrm{SL}_{2} / \mathbb{Z}_{3}\right] \otimes_{K} K\left[z, z^{-1}\right]$, 
а это следует из

$$
K[X]_{(z)} \cong K\left[\frac{a}{z}, \frac{b}{z}, \frac{c}{z}, \frac{d}{z}\right] \otimes_{K} K\left[z, z^{-1}\right]
$$

Особьй слой многообразия $X$ изоморфен нуль-конусу в $V_{3}$. Этот нуль-конус неприводим, содержит плотную орбиту $\mathrm{SL}_{2}\left(x^{2} y\right)$ и двумерную орбиту $\mathrm{SL}_{2}\left(x^{3}\right)$. Несложно проверить, что точки последней орбиты особы в нуль-конусе, и потому нуль-конус не нормален. Это доказывает, что многообразие $X$ не может быть получено как тотальное пространство стягивания относительно некоторой фильтрации на алгебре $K\left[\mathrm{SL}_{2} / \mathbb{Z}_{3}\right]$, определенной отображением $\varphi: \Xi(Y) \rightarrow \mathbb{Z}$.

11. В этом разделе будут рассмотрены редуктивные алгебраические полугруппы. Мы кратко опишем, как теорема 1 согласуется с результатами работы [6].

Напомним, что аффинная алгебраическая полугруппа - это аффинное алгебраическое многообразие с ассоциативным умножением

$$
\sigma: S \times S \rightarrow S
$$

которое есть морфизм алгебраических многообразий.

Пусть End $V$ - кольцо эндоморфизмов конечномерного векторного пространства $V$. Можно показать, что каждая алгебраическая полугруппа изоморфна замкнутой подполугруппе в End $V$ при подходящем выборе $V$. Более того, если полугруппа $S$ обладает единицей, то можно считать, что она (единица) переходит в единицу End $V$. В этой ситуации элемент из $S$ обратим тогда и только тогда, когда он принадлежит $\mathrm{GL}(V)$. Поэтому группа обратимых элементов $G(S)$ открыта в $S$ и является алгебраической группй. Полугруппа $S$ назьвается редуктивной, если группа $G(S)$ редуктивна. Известно, что если группа $G(S)$ полупроста, то $S=G(S)$. Полугруппа $S$ называется нормальной, если $S$ нормально как алгебраическое многообразие.

В работе [6] Э. Б. Винберг классифищировал нормальные редуктивные алгебраические полугруппы в терминах выпуклых конусов в пространстве $\Xi(G(S)) \otimes_{\mathbb{Z}} \mathbb{Q}$. Заметим, что редуктивная группа $G$ есть однородное сферическое пространство относительно двухстороннего действия группы $G \times G$. Алгебра регулярных функций $K[G]$ как $(G \times G)$-модуль разлагается в прямую сумму подмодулей $V_{\lambda} \otimes V_{\lambda^{\prime}}$, где первый сомножитель есть неприводимый $G$-модуль со старшим весом $\lambda$ относительно левого действия, а второй множитель - сопряженньй неприводимый модуль относительно правого $G$-действия. Веса $\lambda$ пробегают всю полугруппу $\Xi_{+}(G)$. Это позволяет строить соответствуюшие конусы в пространстве $\Xi(G(S)) \otimes_{\mathbb{Z}} \mathbb{Q}$, а не в пространстве $\Xi(G(S) \times G(S)) \otimes_{\mathbb{Z}} \mathbb{Q}$.

Тот факт, что $G$ как однородное $(G \times G)$-пространство относится к типу I (см. п. 6) , вытекает из следующей леммы.

Лемма 4 [6; п. 1]. Пусть $\lambda \in \Xi_{+}(G)$ и $\alpha$ - простой корень такой, что $(\lambda, \alpha)>0$. Tогда $2 \lambda-\alpha \in T_{G}(\lambda, \lambda)$.

Тем самьм задача построения конусов стягивания здесь решается вполне механически. 
Отметим, что редуктивную алгебраическую полугруппу $S$ можно рассматривать как многопараметрическую деформацию максимальной полупростой подгруппы $G_{0}$ группы $G(S)$, см. [6; теорема 3] (число параметров равно размерности центра $G(S)$ ). Поэтому конусы из работы [6] суть конусы многомерного стягивания.

Известно, что изотипную компоненту $W_{\lambda}$ в алгебре $K[G]$ можно реализовать как векторное пространство, порожденное матричными элементами неприводимого представления групшы $G$ со старшим весом $\lambda$. Умножению в $G$ соответствует коумножение в $K[G]$ :

$$
K[G] \rightarrow K[G] \otimes K[G], \quad f_{i, j}^{\lambda} \rightarrow \sum_{k} f_{i, k}^{\lambda} \otimes f_{k, j}^{\lambda}
$$

Для каждого $(G \times G)$-многообразия с открытой орбитой, изоморфной $G$, алгебра регулярных функций есть $(G \times G)$-инвариантная подалгебра в $K[G]$. Поэтому каждое такое многообразие имеет естественную структуру редуктивной алгебраической полугруппы с группой обратимых элементов $G$. В частности, $\left(G \times G, G, \mathbb{A}^{1}\right)$-многообразие есть редуктивная алгебраическая полугруппа с группой обратимых элементов $G \times K^{*}$.

Обратно, если $G_{0}$ есть связная полупростая группа, то каждая нормальная полугруппа $S$ с $G(S)=G_{0} \times K^{*}$ есть $\left(G_{0} \times G_{0}, G_{0}, \mathbb{A}^{1}\right)$-многообразие (см. [6; теоремa 3]).

Итак, в однопараметрическом случае имеет место

ПреДЛОЖЕНИЕ 6. Пусть $G_{0}$ - связная полупростая группа. Тогда нормальные редуктивные алгебраические полугруппь с группой обратимых әлементов $G_{0} \times K^{*}$ (кроме самой группьи $\left.G_{0} \times K^{*}\right)$ находятся в биективном соответствии с нормальными $\left(G_{0} \times G_{0}\right)$-инвариантными фильтрациями на алгебре $K\left[G_{0}\right]$.

12. Проиллюстрируем понятие конуса стягивания на примере аффинных трехмерных $\mathrm{SL}_{2}$-многообразий.

Зафиксируем следующие обозначения. Пусть $T$ есть максимальный тор в группе $\mathrm{SL}_{2}, N$ обозначает нормализатор максимального тора $T$ и

$$
U_{d}=\left\{\left(\begin{array}{cc}
\xi & a \\
0 & \xi^{-1}
\end{array}\right): a, \xi \in K, \xi^{d}=1\right\}, \quad d=1,2,3 \ldots
$$

есть конечное расширение максимальной унипотентной подгруппы $U$. Пусть $\varepsilon$ есть фундаментальный вес группы $\mathrm{SL}_{2}$ и $V_{d}-\mathrm{SL}_{2}$-модуль бинарных форм степени $d$.

Аффинное сферическое многообразие групшы $\mathrm{SL}_{2}$ изоморфно либо замыканию орбиты старшего вектора в неприводимом представлении $\mathrm{SL}_{2}$ в пространстве $V_{d}$ (открытая орбита в таком замыкании есть $\mathrm{SL}_{2} / U_{d}$ ), либо одному из однородных пространств $\mathrm{SL}_{2} / N$ или $\mathrm{SL}_{2} / T$. Соответствующие этим сферическим многообразиям $\left(\mathrm{SL}_{2}, Y, \mathbb{A}^{1}\right)$-многообразия будем называть простьми $(S, U)-,(S, N)$ - и $(S, T)$-многообразиями соответственно, см. [10]. 


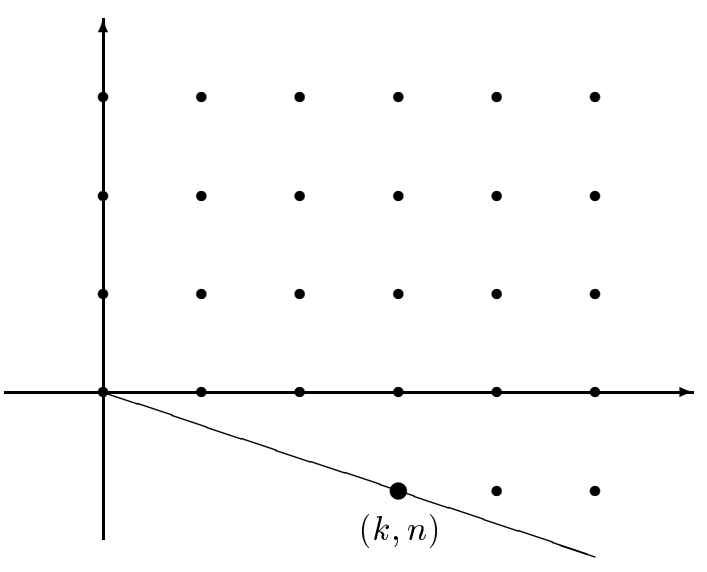

Рис. 3

(1) Простые $(S, U)$-многообразия. Если $Y$ есть вложение однородного пространства $\mathrm{SL}_{2} / U_{d}$, то $\Xi(Y)=\{0, d \varepsilon, 2 d \varepsilon, \ldots\}$. Здесь $Y$ является $S$-многообразием, поэтому выполнено условие $(\mathrm{C})$. В качестве конуса стягивания $C$ подходит любой конус того же вида, что и на рис. 3 , где $k$ и $n-$ взаимно простые целые числа и $k>0$ (координате $k$ на горизонтальной оси отвечает вес $k d \varepsilon$ ).

Задача о построении простого $(S, U)$-многообразия $X_{n}^{k}(U)$ сводится к задаче о построении двумерного аффинного торического многообразия $P_{n}^{k}$, веса которого лежат в том же конусе, поскольку

$$
X_{n}^{k}(U)=\left(\mathbb{A}^{2} \times P_{n}^{k}\right) / / K^{*},
$$

где группа $\mathrm{SL}_{2}$ действует только на первом множителе, а тор $K^{*}$ действует на $\mathbb{A}^{2}$ скалярно и на алгебре $K\left[P_{n}^{k}\right]$ с весами, соответствующими горизонтальной оси.

Рассмотрим два примера.

1) Целочисленные стягивания отвечают $k=1$. При $d=1$ мы получаем единственное с точностью до $\mathrm{SL}_{2}$-изоморфизма гладкое простое $(S, U)$-многообразие это аффинное пространство $\mathbb{A}^{3}=V_{0} \oplus V_{1}$ ( $n$ можно выбирать произвольно). При $d>1$ соответствуюшее многообразие изоморфно $\mathbb{A}^{3} / \mathbb{Z}_{d}$.

2) Пусть $d=1, k=2, n=-1$. Тогда образуюшие алгебры $K\left[P_{-1}^{2}\right]$ обозначим через $x(0,1), y(1,0)$ и $z(2,-1)$. Они связаны соотношением $y^{2}=x z$. Данное торическое многообразие есть конус в $\mathbb{A}^{3}$. Обозначим через $u$ и $v$ стандартные координаты на $\mathbb{A}^{2}$. Для простого $(S, U)$-многообразия получаем $K\left[X_{-1}^{2}(U)\right]=K[x, y, z, u, v]^{K^{*}}=K\left[x_{1}, x_{2}, x_{3}, x_{4}, x_{5}, x_{6}\right]$, где $x_{1}=x, x_{2}=y u$, $x_{3}=y v, x_{4}=z u^{2}, x_{5}=z u v, x_{6}=z v^{2}$ и выполнены соотношения $x_{2}^{2}=x_{1} x_{4}$, $x_{3}^{2}=x_{1} x_{6}, x_{2} x_{3}=x_{1} x_{5}, x_{4} x_{6}=x_{5}^{2}$. Это многообразие естественно вложено в пространство $\mathbb{A}^{6}=V_{0} \oplus V_{1} \oplus V_{2}$, не является там полным пересечением и имеет единственную особенность в нуле.

ПРЕДЛОЖЕНИЕ 7. При фиксированном $d$ nростые $(S, U)$-многообразия $X_{n}^{k}(U)$ и $X_{n^{\prime}}^{k^{\prime}}(U) \mathrm{SL}_{2}$-әквивариантно изоморфньи тогда и только тогда, когда $k=k^{\prime}$ un $\equiv n^{\prime}(\bmod k)$. 
ДокАЗАТЕЛЬСтво. Заметим, что наличие $\mathrm{SL}_{2}$-эквивариатного изоморфизма между $X_{n}^{k}(U)$ и $X_{n^{\prime}}^{k^{\prime}}(U)$ эквивалентно наличию $K^{*}$-эквивариантного изоморфизма между $P_{n}^{k}$ и $P_{n^{\prime}}^{k^{\prime}}$, где $K^{*}$ - одномерный тор, веса которого мы откладываем по горизонтальной оси. Это вытекает из формулы (***) и того факта, что $\operatorname{Spec} K\left[X_{n}^{k}(U)\right]^{U} \cong P_{n}^{k}$. Поэтому доказательство сводится к рассмотрению двумерных торических многообразий.

Достаточность. Реализуем алгебру $K\left[P_{n}^{k}\right]$ как подалгебру в алгебре многочленов $K[X, Y]$ с действием тора $K^{*}$ по формуле $X \rightarrow t X, Y \rightarrow Y$ :

$$
K\left[P_{n}^{k}\right]=\left\langle X^{i} Y^{j}:(i, j) \in C\right\rangle
$$

Пусть $n-n^{\prime}=m k$. Тогда требуемьй изоморфизм задается формулой $X^{i} Y^{j} \rightarrow$ $X^{i} Y^{j+m i}$.

Необходимость. Равенство $k=k^{\prime}$ следует из того, что плотная орбита в особом слое изоморфна $\mathrm{SL}_{2} / U_{d k}$. Предположим теперь, что $n, n^{\prime} \in[0, k-1]$. Соединим ломаной точки с целыми координатами в конусе $C$, отвечающие таким регулярньм функциям, которые при сужении на особый слой становятся ненулевыми нильпотентами весов $1,2, \ldots, k-1$, см. рис. 4 .

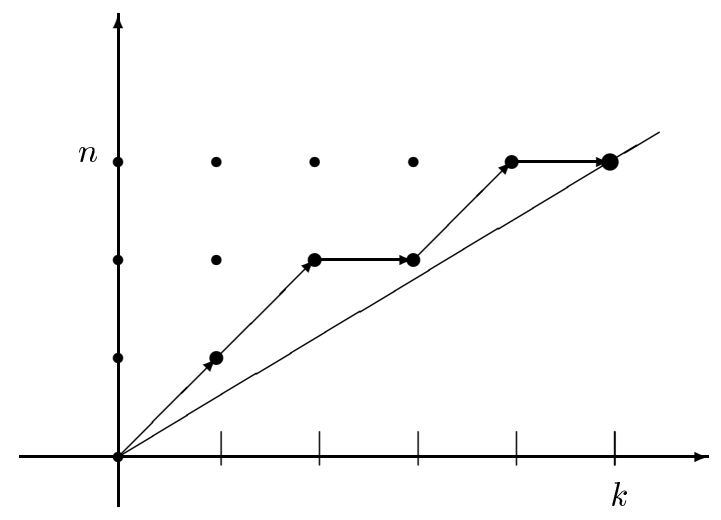

Рис. 4

Горизонтальное звено такой ломаной $(l, m) \rightarrow(l+1, m)$ соответствует тому, что произведение нильпотентов веса 1 и $l$ равно нулю, а звено $(l, m) \rightarrow(l+1, m+1)$ означает, что это произведение есть ненулевой нильпотент. Поэтому если ломаные различны, то многообразия неизоморфны. Но при $n \neq n^{\prime}$ ломаные не могут совпадать, так как их высоты равны $n$ и $n^{\prime}$ соответственно.

(2) Простые $(S, N)$-многообразия. Здесь $Y=\mathrm{SL}_{2} / N, \Xi(Y)=\{0,4 \varepsilon, 8 \varepsilon, \ldots\}$ и

$$
T_{Y}(4 a \varepsilon, 4 b \varepsilon)=\{4(a+b) \varepsilon, 4(a+b-1) \varepsilon, \ldots, 4(a-b) \varepsilon \text { при } a \geqslant b\} .
$$

Условие 3 ) из определения конуса стягивания означает, что в качестве конуса стягивания здесь подходит любой конус того же вида, что и на рис. 5 , где $k$ и $n-$ взаимно простые целые числа и $k>0, n \geqslant 0$. 


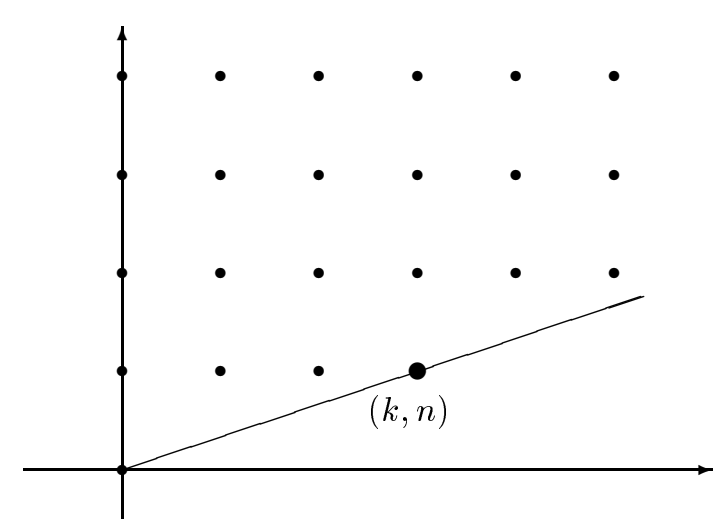

Рис. 5

Приведем с некоторьми усовершенствованиями конструкцию из работы [10], peализуюшую простые $(S, N)$-многообразия для всех значений $k$ и $n$. Пусть $a, b, c$ - стандартные координаты в пространстве квадратичных бинарных форм $V_{2}=$ $\left\{a x^{2}+b x y+c y^{2}\right\}$. Рассмотрим фактормногообразие $X^{k}=V_{2} / \mathbb{Z}_{2 k}$, где циклическая групша $\mathbb{Z}_{2 k}$ действует на векторном пространстве $V_{2}$ скалярно умножениями на корни степени $2 k$ из единицы, и пусть $X_{n}^{k}(N)$ есть $\mathrm{SL}_{2}$-многообразие, заданное в многообразии $X^{k} \times \mathbb{A}^{1}$ уравнением $z^{n}=\left(b^{2}-4 a c\right)^{k}$ (предполагается, что координата $z$ инвариантна при действии группы $\mathrm{SL}_{2}$ ). При действии тора $K^{*}$ переменная $z$ имеет вес 1 , а каждый моном от переменных $a, b, c$ степени $2 k$ имеет вес $n$.

Многообразие $X_{n}^{k}(N)$ нормально только при $n=1$ или $k=1$, см. [10]. В любом случае его нормализация $\operatorname{Norm} X_{n}^{k}(N)$ является простым $(S, N)$-многообразием, соответствуюшим паре $(k, n)$. Опишем явно эту нормализацию. Будем обозначать через $M_{d}$ множество всех мономов степени $d$ от переменных $a, b, c$. Пусть $u$ и $v$ - такие натуральные числа, что $n v-k u=1$. Обозначим через $\Pi(k, n)$ параллелограмм в $\mathbb{A}^{2}$ с вершиной в нуле, натянутый на векторы $(k, n)$ и $(0,1)$. Множество целых точек этого параллелограмма есть множество образуюших полугруппы целых точек конуса стягивания. Отсюда следует, что

$$
K\left[\operatorname{Norm} X_{n}^{k}(N)\right]=K\left[X_{n}^{k}(N)\right]\left[\frac{M_{2 k_{1}}\left(b^{2}-4 a c\right)^{\left(k n_{1}-n k_{1}\right) v}}{z^{\left(k n_{1}-n k_{1}\right) u}}\right],
$$

где пары $\left(k_{1}, n_{1}\right)$ пробегают координаты целых точек внутренности параллелограмма $\Pi(k, n)$.

В работе [10] доказано, что морфизм нормализации $\operatorname{Norm} X_{n}^{k}(N) \rightarrow X_{n}^{k}(N)$ биективен и простые $(S, N)$-многообразия, соответствуюшие различньм парам $(k, n)$, не являются $\mathrm{SL}_{2}$-изоморфньми.

(3) Простые $(S, T)$-многообразия. Здесь $Y=\mathrm{SL}_{2} / T, \Xi(Y)=\{0,2 \varepsilon, 4 \varepsilon, \ldots\}$ и

$$
T_{Y}(2 a \varepsilon, 2 b \varepsilon)=\{2(a+b) \varepsilon, 2(a+b-1) \varepsilon, \ldots, 2(a-b) \varepsilon \text { при } a \geqslant b\} .
$$

Условие 3 ) из определения конуса стягивания означает, что в качестве конуса стягивания здесь подходит любой конус того же вида, что и на рис. 5. 
Однородное пространство $\mathrm{SL}_{2} / T$ допускает единственный нетривиальный эквивариантный автоморфизм (порядка два), фактор по которому есть $\mathrm{SL}_{2} / N$. Несложно показать, что действие этого автоморфизма продолжается до действия на всяком простом $(S, T)$-многообразии, соответствующем паре $(k, n)$, и в качестве фактора получается простое $(S, N)$-многообразие, отвечающее паре $(k / 2, n)$ для четного $k$ и паре $(k, 2 n)$ для нечетного $k$. Отображение факторизации есть двулистное накрытие, разветвленное над особым слоем в первом случае и над одной точкой во втором. Отсюда следует, что простые $(S, T)$-многообразия, отвечающие различным парам $(k, n)$, не являются $\mathrm{SL}_{2}$-изоморфньми.

В случае нечетного $k$ можно построить простое $(S, T)$-многообразие так же, как и простое $(S, N)$-многообразие. Рассмотрим фактормногообразие $X^{k}=V_{2} / \mathbb{Z}_{k}$, и пусть $X_{n}^{k}(T)$ есть $\mathrm{SL}_{2}$-многообразие, заданное в многообразии $X^{k} \times \mathbb{A}^{1}$ уравнением $z^{2 n}=\left(b^{2}-4 a c\right)^{k}$. При действии тора $K^{*}$ переменная $z$ имеет вес 1 , а каждый моном от переменных $a, b, c$ степени $k$ имеет вес $n$.

Пусть $u$ и $v$ - такие натуральные числа, что $2 n v-k u=1$. Тогда

$$
K\left[\operatorname{Norm} X_{n}^{k}(T)\right]=K\left[X_{n}^{k}(T)\right]\left[\frac{M_{k_{1}}\left(b^{2}-4 a c\right)^{\left(k n_{1}-n k_{1}\right) v}}{z^{\left(k n_{1}-n k_{1}\right) u}}\right]
$$

где пары $\left(k_{1}, n_{1}\right)$ пробегают координаты целых точек внутренности параллелограмма $\Pi(k, n)$.

Целочисленным стягиваниям здесь отвечают гиперповерхности в четырехмерном пространстве.

Для четных значений $k$ такую реализацию получить нельзя. В этом случае (как и в случае нечетного $k$ ) простое $(S, T)$-многообразие можно реализовать либо как двулистное накрытие соответствуюшего простого $(S, N)$-многообразия, либо следующим образом. Рассмотрим многообразие $X_{n}^{1}(T)$ с координатами $(z, a, b, c)$ и многообразие $X^{k}$, соответствующее координатам $\left(z^{\prime}, a^{\prime}, b^{\prime}, c^{\prime}\right)$. Тогда искомое Norm $X_{n}^{k}(T)$ получается нормализацией подмногообразия в $X_{n}^{1}(T) \times X^{k}$, заданного уравнениями $z=z^{\prime}, w(a, b, c)=z^{n(k-1)} w\left(a^{\prime}, b^{\prime}, c^{\prime}\right)$ для каждого монома $w \in M_{k}$.

\section{Список литературы}

1. Попов В. Л. Стягивание действий редуктивных алгебраических групп // Матем. сб. 1986. Т. 130 (172). № 3 (7). С. 310-334.

2. Аржаниев И. В. О действиях редуктивных групп с однопараметрическим семейством сферических орбит // Матем. сб. 1997. Т. 188. № 5. С. 3-20.

3. Luna D., Vust Th. Plongements d'espaces homogènes // Comment. Math. Helv. 1983. V. 58. P. $186-245$.

4. Винберг Э. Б., Попов В. Л. Об одном классе квазиоднородных аффиинных многообразий // Изв. АН СССР. Сер. матем. 1972. Т. 36. № 4. С. 749-764.

5. Попов $В$. Л. Квазиоднородные афффинные алгебраические многообразия группы $\mathrm{SL}(2)$ // Изв. АН СССР. Сер. матем. 1973. Т. 37. № 4. С. 792-832.

6. Vinberg E. B. On reductive algebraic semigroups // Amer. Math. Soc. Transl. Ser. 2. 1995. V. 169. P. 145-182.

7. Крафm X. Геометрические методы в теории инвариантов. М.: Мир, 1987.

8. Данилов В.И. Геометрия торических многообразий // УМН. 1978. Т. 32. № 2. C. $85-134$. 
9. Винберг Э. Б., Попов В. Л. Теория инвариантов // Итоги науки и техн. Совр. пробл. матем. Фундам. напр. Т. 55. М.: ВИНИТИ, 1989. С. 137-314.

10. Аржсанцев И. В. О действиях сложности один группы $\mathrm{SL}_{2} / /$ Изв. РАН. Сер. матем. 1997. Т. 61. № 4. С. 3-18.

11. Аржанцев И.В. О нормальности замыканий сферических орбит // Функц. анализ и его прилож. 1997. Т. 31. № 4. С. 66-69.

Московский государственньй

Поступила в редакцию

университет им. М.В. Ломоносова

06.08.1998

E-mail : arjantse@mccme.ru 